\title{
STOCHASTIC EQUATIONS WITH BOUNDARY NOISE
}

\author{
ROLAND SCHNAUBELT AND MARK VERAAR
}

Abstract. We study the wellposedness and pathwise regularity of semilinear non-autonomous parabolic evolution equations with boundary and interior noise in an $L^{p}$ setting. We obtain existence and uniqueness of mild and weak solutions. The boundary noise term is reformulated as a perturbation of a stochastic evolution equation with values in extrapolation spaces.

\section{INTRODUCTION}

In this paper we investigate the wellposedness and pathwise regularity of semilinear non-autonomous parabolic evolution equations with boundary noise. A model example which fits in the class of problems we study is given by

$$
\begin{array}{rlrl}
\frac{\partial u}{\partial t}(t, s) & =\mathcal{A}(t, s, D) u(t, s) & \text { on }(0, T] \times S, \\
\mathcal{B}(t, s, D) u(t, s) & =c(t, u(t, s)) \frac{\partial w}{\partial t}(t, s) & & \text { on }(0, T] \times \partial S, \\
u(0, s) & =u_{0}(s), \quad & & \text { on } S .
\end{array}
$$

Here $S \subset \mathbb{R}^{d}$ is a bounded domain with $C^{2}$ boundary, $\mathcal{A}(t, \cdot, D)=\operatorname{div}(a(t, \cdot) \nabla)$ for uniformly positive definite, symmetric matrices $a(t, s)$ with the conormal boundary operator $\mathcal{B}(t, s, D), c(t, \xi)$ is Lipschitz in $\xi \in \mathbb{C},(w(t))_{t \geq 0}$ is a Brownian motion for an filtration $\left\{\mathcal{F}_{t}\right\}_{t \geq 0}$ and with values in $L^{r}(\partial S)$ for some $r \geq 2$, and $u_{0}$ is an $\mathcal{F}_{0}$-measurable initial value. Actually, we also allow for lower order terms, interior noise, nonlocal nonlinearities, and more general stochastic terms, see Section 4.

As a first step one has to give a precise meaning to the formal boundary condition in (1.1). We present two solution concepts for (1.1) in Section 4, namely a mild and a weak one, which are shown to be equivalent. Our analysis is then based on the mild version of (1.1), which fits into the general framework of [29] where parabolic non-autonomous evolution equations in Banach spaces were treated. The results in [29] rely on the stochastic integration theory in certain classes of Banach spaces (see $[8,21,23])$. In order to use [29], the inhomogeneous boundary term is reformulated as an additive perturbation of a stochastic evolution equation corresponding to homogeneous boundary conditions. This perturbation maps into a so-called extrapolation space for the realization $A(t)$ of $\mathcal{A}(t, \cdot, D)$ in $L^{p}(S)$ with the boundary condition $\mathcal{B}(t, \cdot, D) u=0$ (where $p \in[2, r]$ ). Such an approach was developed for deterministic problems by Amann in e.g. [5] and [6]. We partly use somewhat different techniques taken from [18], see also the references therein. For this reformulation, one further needs the solution map of a corresponding elliptic boundary value problem with boundary data in $L^{r}(\partial S)$ which is the range space of the Brownian motion. Here we heavily rely on the theory presented in [5], see also the references therein. We observe that in [5] a large class of elliptic systems was

2000 Mathematics Subject Classification. Primary 60H15; Secondary 35R60, 47D06.

Key words and phrases. Parabolic stochastic evolution equation, multiplicative boundary noise, non-autonomous equations, mild solution, variational solution, extrapolation.

The second named author was supported by the Alexander von Humboldt foundation and by a VENI subsidie (639.031.930) in the Vernieuwingsimpuls programme of the Netherlands Organization for Scientific Research (NWO). 
studied. Accordingly, we could in fact allow for systems in (1.1), but we decided to restrict ourselves to the scalar case in order to simplify the presentation.

We establish in Theorem 4.3 the existence and uniqueness of a mild solution $u$ to (1.1). Such a solution is a process $u:[0, T] \times \Omega \rightarrow L^{p}(S)$ where $(\Omega, P)$ is the probability space for the Brownian motion. We further show that for a.e. fixed $\omega \in \Omega$ the path $t \mapsto u(t, \omega)$ is (Hölder) continuous with values in suitable interpolation spaces between $L^{p}(S)$ and the domain of $A(t)$, provided that $u_{0}$ belongs to a corresponding interpolation space a.s.. As a consequence, the paths of $u$ belong to $C\left([0, T], L^{q}(S)\right)$ for all $q<d p /(d-1)$. At this point, we make use of the additional regularity provided by the $L^{p}$ approach to stochastic evolution equations.

In [20] an autonomous version of (1.1) has been studied in a Hilbert space situation (i.e., $r=p=2$ ) employing related techniques. However, in this paper only regularity in the mean and no pathwise regularity has been treated. In [12, §13.3], Da Prato and Zabczyk have also investigated boundary noise of Neumann type. They deal with a specific situation where $a(t)=I$, the domain is a cube and the noise acts on one face which allows more detailed results. See also [3], [13] and [27] for further contributions to problems with boundary noise. As explained in Remark 4.9 we cannot treat Dirichlet type boundary conditions due to our methods. In one space dimension Dirichlet boundary noise has been considered in [4] in weighted $L^{p}$-spaces by completely different techniques.

In the next section, we first recall the necessary material about parabolic deterministic evolution equations and about stochastic integration. Then we study an abstract stochastic evolution equation related to (1.1) in Section 3. Finally, in the last section we treat a more general version of (1.1) and discuss various examples concerning the stochastic terms.

\section{Preliminaries}

We write $a \lesssim_{K} b$ if there exists a constant $c$ only depending on $K$ such that $a \leq c b$. The relation $a \bar{\sim}_{K} b$ expresses that $a \lesssim_{K} b$ and $b \lesssim_{K} a$. If it is clear what is meant, we just write $a \lesssim b$ for convenience. Throughout, $X$ denotes a Banach space, $X^{*}$ its dual, and $\mathcal{B}(X, Y)$ the space of linear bounded operators from $X$ into another Banach space $Y$. If the spaces are real, everything below should be understood for the complexification of the objects under consideration. The complex interpolation space for an interpolation couple $\left(X_{1}, X_{2}\right)$ of order $\eta \in(0,1)$ is designated by $\left[X_{1}, X_{2}\right]_{\eta}$. We refer to [28] for the relevant definitions and basic properties.

2.1. Parabolic evolution families. We briefly discuss the approach to non-autonomous parabolic evolution equations developed by Acquistapace and Terreni, [2]. For $w \in \mathbb{R}$ and $\phi \in[0, \pi]$, set $\Sigma(\phi, w)=\{\lambda \in \mathbb{C}:|\arg (z-w)| \leq \phi\}$. A family $(A(t), D(A(t)))_{t \in[0, T]}$ satisfies the hypothesis (AT) if the following two conditions hold, where $T>0$ is given.

(AT1) $A(t)$ are densely defined, closed linear operators on a Banach space $X$ and there are constants $w \in \mathbb{R}, K \geq 0$, and $\phi \in\left(\frac{\pi}{2}, \pi\right)$ such that $\Sigma(\phi, w) \subset$ $\varrho(A(t))$ and

$$
\|R(\lambda, A(t))\| \leq \frac{K}{1+|\lambda-w|}
$$

holds for all $\lambda \in \Sigma(\phi, w)$ and $t \in[0, T]$.

(AT2) There are constants $L \geq 0$ and $\mu, \nu \in(0,1]$ such that $\mu+\nu>1$ and

$$
\left\|A_{w}(t) R\left(\lambda, A_{w}(t)\right)\left(A_{w}(t)^{-1}-A_{w}(s)^{-1}\right)\right\| \leq L|t-s|^{\mu}(|\lambda|+1)^{-\nu}
$$

holds for all $\lambda \in \Sigma(\phi, 0)$ and $s, t \in[0, T]$, where $A_{w}(t)=A(t)-w$. 
Condition (A1) just means sectoriality with angle $\phi>\pi / 2$ and uniform constants, whereas (A2) says that the resolvents satisfy a Hölder condition in stronger norms. In fact, Acquistapace and Terreni have studied a somewhat weaker version of (AT2) and allowed for non dense domains. Later on, we work on reflexive Banach spaces, where sectorial operators are automatically densely defined so that we have included the density assumption in (AT1) for simplicity. The conditions (AT) and several variants of them have intensively been studied in the literature, where also many examples can be found, see e.g. [1, 2, 6, 25, 30]. If (AT1) holds and the domains $D(A(t))$ are constant in time, then the Hölder continuity of $A(\cdot)$ in $\mathcal{B}(D(A(0)), X)$ with exponent $\eta$ implies (AT2) with $\mu=\eta$ and $\nu=1$ (see [2, Section 7]).

Let $\eta \in(0,1), \theta \in[0,1]$, and $t \in[0, T]$. Assume that (AT1) holds. The fractional power $\left(-A_{w}(t)\right)^{-\theta} \in \mathcal{B}(X)$ is defined by

$$
\left(-A_{w}(t)\right)^{-\theta}=\frac{1}{2 \pi i} \int_{\Gamma}(w-\lambda)^{-\theta} R(\lambda, A(t)) d \lambda,
$$

where the contour $\Gamma=\{\lambda: \arg (\lambda-w)= \pm \phi\}$ is orientated counter clockwise. The operator $(w-A(t))^{\theta}$ is defined as the inverse of $(w-A(t))^{-\theta}$. We will also use the complex interpolation space

$$
X_{\eta}^{t}=[X, D(A(t))]_{\eta} .
$$

Moreover, the extrapolation space $X_{-\theta}^{t}$ is the completion of $X$ with respect to the norm $\|x\|_{X_{-\theta}^{t}}=\left\|\left(-A_{w}(t)\right)^{-\theta} x\right\|$. Let $A_{-1}(t): X \rightarrow X_{-1}^{t}$ be the unique continuous extension of $A(t)$ which is sectorial of the same type. Then $\left(w-A_{-1}(t)\right)^{\alpha}: X_{-\theta}^{t} \rightarrow$ $X_{-\theta-\alpha}^{t}$ is an isomorphism, where $0 \leq \theta \leq \alpha+\theta \leq 1$. If $X$ is reflexive, then one can identify the dual space $\left(X_{-1}^{t}\right)^{*}$ with $D\left(A(t)^{*}\right)$ endowed with its graph norm and the adjoint operator $A_{-1}(t)^{*}$ with $A(t)^{*} \in \mathcal{B}\left(D\left(A(t)^{*}\right), X^{*}\right)$. We mostly write $A(t)$ instead of $A_{-1}(t)$. See e.g. [6, 18] for more details.

Under condition (AT), we consider the non-autonomous Cauchy problem

$$
\begin{aligned}
u^{\prime}(t) & =A(t) u(t), \quad t \in[s, T], \\
u(s) & =x
\end{aligned}
$$

for given $x \in X$ and $s \in[0, T)$. A function $u$ is a classical solution of (2.1) if $u \in C([s, T] ; X) \cap C^{1}((s, T] ; X), u(t) \in D(A(t))$ for all $t \in(s, T], u(s)=x$, and $\frac{d u}{d t}(t)=A(t) u(t)$ for all $t \in(s, T]$. The solution operators of (2.1) give rise to the following definition. A family of bounded operators $(P(t, s))_{0 \leq s \leq t \leq T}$ on $X$ is called a strongly continuous evolution family if

(1) $P(s, s)=I$ for all $s \in[0, T]$,

(2) $P(t, s)=P(t, r) P(r, s)$ for all $0 \leq s \leq r \leq t \leq T$,

(3) the map $\left\{(\tau, \sigma) \in[0, T]^{2}: \sigma \leq \tau\right\} \ni(t, s) \rightarrow P(t, s)$ is strongly continuous.

The next theorem says that the operators $A(t), 0 \leq t \leq T$, 'generate' an evolution family having parabolic regularity. It is a consequence of [1, Theorem 2.3], see also $[2,6,25,30]$.

Theorem 2.1. If condition (AT) holds, then there exists a unique strongly continuous evolution family $(P(t, s))_{0 \leq s \leq t \leq T}$ such that $u=P(\cdot, s) x$ is the unique classical solution of (2.1) for every $x \in \bar{X}$ and $s \in[0, T)$. Moreover, $(P(t, s))_{0 \leq s \leq t \leq T}$ is continuous in $\mathcal{B}(X)$ on $0 \leq s<t \leq T$ and there exists a constant $C>0$ such that

$$
\|P(t, s) x\|_{X_{\alpha}^{t}} \leq C(t-s)^{\beta-\alpha}\|x\|_{X_{\beta}^{s}}
$$

for all $0 \leq \beta \leq \alpha \leq 1$ and $0 \leq s<t \leq T$.

We further recall from [31, Theorem 2.1] that there is a constant $C>0$ such that

$$
\left\|P(t, s)(w-A(s))^{\theta} x\right\| \leq C(\mu-\theta)^{-1}(t-s)^{-\theta}\|x\|
$$


for all $0 \leq s<t \leq T, \theta \in(0, \mu)$ and $x \in D\left((w-A(s))^{\theta}\right)$. Clearly, (2.3) allows to extend $P(t, s)$ to a bounded operator $P_{-\theta}(t, s): X_{-\theta}^{s} \rightarrow X$ satisfying

$$
\left\|P_{-\theta}(t, s)\left(w-A_{-1}(s)\right)^{\theta}\right\| \leq C(\mu-\theta)^{-1}(t-s)^{-\theta}
$$

for all $0 \leq s<t \leq T$ and $\theta \in(0, \mu)$. Again, we mostly omit the index $-\theta$.

2.2. Stochastic integration. Let $H$ be a separable Hilbert space with scalar product $[\cdot, \cdot], X$ be a Banach space, and $(S, \Sigma, \mu)$ be a measure space. A function $\phi: S \rightarrow X$ is called strongly measurable if it is the pointwise limit of a sequence of simple functions. Let $X_{1}$ and $X_{2}$ be Banach spaces. An operator-valued function $\Phi: S \rightarrow \mathcal{B}\left(X_{1}, X_{2}\right)$ will be called $X_{1}$-strongly measurable if the $X_{2}$-valued function $\Phi x$ is strongly measurable for all $x \in X_{1}$.

Throughout this paper $(\Omega, \mathcal{F}, \mathbb{P})$ is a probability space with a filtration $\left(\mathcal{F}_{t}\right)_{t \geq 0}$ and $\left(\gamma_{n}\right)_{n \geq 1}$ is a Gaussian sequence; i.e., a sequence of independent, standard, realvalued Gaussian random variables defined on $(\Omega, \mathcal{F}, \mathbb{P})$. An operator $R \in \mathcal{B}(H, X)$ is said to be a $\gamma$-radonifying operator if there exists an orthonormal basis $\left(h_{n}\right)_{n \geq 1}$ of $H$ such that $\sum_{n \geq 1} \gamma_{n} R h_{n}$ converges in $L^{2}(\Omega ; X)$, see [7, 16]. In this case we define

$$
\|R\|_{\gamma(H, X)}:=\left(\mathbb{E}\left\|\sum_{n \geq 1} \gamma_{n} R h_{n}\right\|^{2}\right)^{\frac{1}{2}} .
$$

This number does not depend on the sequence $\left(\gamma_{n}\right)_{n \geq 1}$ and the basis $\left(h_{n}\right)_{n \geq 1}$, and defines a norm on the space $\gamma(H, X)$ of all $\gamma$-radonifying operators from $H$ into $X$. Endowed with this norm, $\gamma(H, X)$ is a Banach space, and it holds $\|R\| \leq\|R\|_{\gamma(H, X)}$. Moreover, $\gamma(\mathcal{H}, X)$ is an operator ideal in the sense that if $S_{1} \in \mathcal{B}(\tilde{\mathcal{H}}, \mathcal{H})$ and $S_{2} \in \mathcal{B}(X, \tilde{X})$, then $R \in \gamma(\mathcal{H}, X)$ implies $S_{2} R S_{1} \in \gamma(\tilde{\mathcal{H}}, \tilde{X})$ and

$$
\left\|S_{2} R S_{1}\right\|_{\gamma(\tilde{\mathcal{H}}, \tilde{X})} \leq\left\|S_{2}\right\|\|R\|_{\gamma(\mathcal{H}, X)}\left\|S_{1}\right\| .
$$

If $X$ is a Hilbert space, then $\gamma(H, X)=\mathcal{C}^{2}(H, X)$ isometrically, where $\mathcal{C}^{2}(H, X)$ is the space of Hilbert-Schmidt operators. Also for $X=L^{p}$ there is a convenient characterization of $R \in \gamma\left(H, L^{p}\right)$ given in [10, Theorem 2.3]. We use a slightly different formulation taken from [22, Lemma 2.1].

Lemma 2.2. Let $(S, \Sigma, \mu)$ be a $\sigma$-finite measure space and let $1 \leq p<\infty$. For an operator $R \in \mathcal{B}\left(H, L^{p}(S)\right)$ the following assertions are equivalent.

(1) $R \in \gamma\left(H, L^{p}(S)\right)$.

(2) There exists a function $g \in L^{p}(S)$ such that for all $h \in H$ we have $|R h| \leq$ $\|h\|_{H} \cdot g \mu$-almost everywhere.

Moreover, in this situation we have

$$
\|R\|_{\gamma\left(H, L^{p}(S)\right)} \lesssim_{p}\|g\|_{L^{p}(S)} .
$$

A Banach space $X$ is said to have type 2 if there exists a constant $C \geq 0$ such that for all finite subsets $\left\{x_{1}, \ldots, x_{N}\right\}$ of $X$ we have

$$
\left(\mathbb{E}\left\|\sum_{n=1}^{N} r_{n} x_{n}\right\|^{2}\right)^{\frac{1}{2}} \leq C\left(\sum_{n=1}^{N}\left\|x_{n}\right\|^{2}\right)^{\frac{1}{2}} .
$$

Hilbert spaces and $L^{p}$-spaces with $p \in[2, \infty)$ have type 2. We refer to [16] for details. We will also need UMD Banach spaces. The definition of a UMD space will be omitted, but we recall that every UMD space is reflexive. We refer to [11] for an overview on the subject. Important examples of UMD spaces are the reflexive scale of $L^{p}$, Sobolev, Bessel-potential and Besov spaces.

A detailed stochastic integration theory for operator-valued processes $\Phi:[0, T] \times$ $\Omega \rightarrow \mathcal{B}(H, X)$, where $X$ is a UMD space, has been developed in [21]. The full generality of this theory is not needed here, since we can work with UMD spaces $X$ of type 2 which allow for a somewhat simpler theory. Instead of of being a UMD 
space with type 2 , one can also assume that $X$ is a space of martingale type 2 (cf. $[8,23])$.

A family $W_{H}=\left(W_{H}(t)\right)_{t \in \mathbb{R}_{+}}$of bounded linear operators from $H$ to $L^{2}(\Omega)$ is called an $H$-cylindrical Brownian motion if

(i) $\left\{W_{H}\left(t_{j}\right) h_{k}: j=1, \ldots, J ; k=1, \ldots, K\right\}$ is a Gaussian vector for all choices of $t_{j} \geq 0$ and $h_{k} \in H$, and $\left\{W_{H}(t) h: t \geq 0\right\}$ is a standard scalar Brownian motion with respect to the filtration $\left(\mathcal{F}_{t}\right)_{t \geq 0}$ for each $h \in H$;

(ii) $\mathbb{E}\left(W_{H}(s) g \cdot W_{H}(t) h\right)=(s \wedge t)[g, h]_{H}$ for all $s, t \in \mathbb{R}_{+}$and $g, h \in H$.

Now let $X$ be a UMD Banach space with type 2. For an $H$-strongly measurable and adapted $\Phi:[0, T] \times \Omega \rightarrow \gamma(H, X)$ which belongs to $L^{2}((0, T) \times \Omega ; \gamma(H, X))$ one can define the stochastic integral $\int_{0}^{T} \Phi(s) d W_{H}(s)$ as a limit of integrals of adapted step processes, and there is a constant $C$ not depending on $\Phi$ such that

$$
\mathbb{E}\left\|\int_{0}^{T} \Phi(s) d W_{H}(s)\right\|^{2} \leq C^{2}\|\Phi\|_{L^{2}((0, T) \times \Omega ; \gamma(H, X))}^{2},
$$

cf. [8], [21], and the references therein. By a localization argument one may extend the class of integrable processes to all $H$-strongly measurable and adapted $\Phi$ : $[0, T] \times \Omega \rightarrow \gamma(H, X)$ which are contained in $L^{2}(0, T ; \gamma(H, X))$ a.s.. Below we use in particular the next result (see [8] and [21, Corollary 3.10]).

Proposition 2.3. Let $X$ be a UMD space with type 2 and $W_{H}$ be a $H$-cylindrical Brownian motion. Let $\Phi:[0, T] \times \Omega \rightarrow \gamma(H, X)$ be $H$-strongly measurable and adapted. If $\Phi \in L^{2}(0, T ; \gamma(H, X))$ a.s., then $\Phi$ is stochastically integrable with respect to $W_{H}$ and for all $p \in(1, \infty)$ it holds

$$
\left(\mathbb{E} \sup _{t \in[0, T]}\left\|\int_{0}^{t} \Phi(s) d W_{H}(s)\right\|^{p}\right)^{\frac{1}{p}} \lesssim_{X, p}\|\Phi\|_{L^{p}\left(\Omega ; L^{2}(0, T ; \gamma(H, X))\right)} .
$$

In the setting of Proposition 2.3 we also have, for $x^{*} \in X^{*}$,

$$
\left\langle\int_{0}^{T} \Phi(s) d W_{H}(s), x^{*}\right\rangle=\int_{0}^{T} \Phi(s)^{*} x^{*} d W_{H}(s) \quad \text { a.s., }
$$

cf. [21, Theorem 5.9].

\section{The abstract StOchastic EVOlution EQUATION}

Let $H_{1}$ and $H_{2}$ be separable Hilbert spaces, and let $X$ and $Y$ be Banach spaces. On $X$ we consider the stochastic evolution equation

$$
\left\{\begin{aligned}
d U(t)= & \left(A(t) U(t)+F(t, U(t))+\Lambda_{G}(t) G(t, U(t))\right) d t \\
& +B(t, U(t)) d W_{H_{1}}(t)+\Lambda_{C}(t) C(t, U(t)) d W_{H_{2}}(t), t \in[0, T], \\
U(0)= & u_{0} .
\end{aligned}\right.
$$

Here $(A(t))_{t \in[0, T]}$ is a family of closed operators on $X$ satisfying (AT). The processes $W_{H_{1}}$ and $W_{H_{2}}$ are independent cylindrical Brownian motions with respect to $\left(\mathcal{F}_{t}\right)_{t \in[0, T]}$. The initial value is a strongly $\mathcal{F}_{0}$-measurable mapping $u_{0}: \Omega \rightarrow X$. We assume that the mappings $\Lambda_{G}(t): Y^{t} \rightarrow X_{-\theta_{G}}^{t}$ and $\Lambda_{C}(t): Y^{t} \rightarrow X_{-\theta_{C}}^{t}$ are linear and bounded, where the numbers $\theta_{G}, \theta_{C} \in[0,1]$ are specified below. In Section 4 , the operators $\Lambda_{G}(t)$ and $\Lambda_{C}(t)$ are used to treat inhomogeneous boundary conditions. Concerning $A(t)$, we make the following hypothesis.

(H1) Assume that $(A(t))_{t \in[0, T]}$ and $\left(A(t)^{*}\right)_{t \in[0, T]}$ satisfy (AT) and that there exists an $\eta_{0} \in(0,1]$ and a family of Banach spaces $\left(\widetilde{X}_{\eta}\right)_{\eta \in\left[0, \eta_{0}\right]}$ such that

$$
\tilde{X}_{\eta_{0}} \hookrightarrow \tilde{X}_{\eta_{1}} \hookrightarrow \tilde{X}_{\eta_{2}} \hookrightarrow \tilde{X}_{0}=X \quad \text { for all } \eta_{0}>\eta_{1}>\eta_{2}>0,
$$


and each $\tilde{X}_{\eta}$ is a UMD space with type 2 . Moreover, it holds

$$
[X, D(A(t))]_{\eta} \hookrightarrow \widetilde{X}_{\eta} \text { for all } \eta \in\left[0, \eta_{0}\right],
$$

where the embeddings are bounded uniformly in $t \in[0, T]$.

Assumption (H1) has been employed in [29] to deduce space time regularity results for equations of the form (SE), where spaces such as $\widetilde{X}_{\eta}$ have been used to get rid of the time dependence of interpolation spaces; see also [19, (H2)]. We have included an assumption on $\left(A(t)^{*}\right)_{t \in[0, T]}$ for the treatment of variational solutions. This could be done in a more general way as well, but for us the above setting suffices. Assumption (H1) can be verified in many applications, see e.g. Section 4.

Let $a \in\left[0, \eta_{0}\right)$. The nonlinear terms $F, G, B$ and $C$ in (SE) map as follows:

$$
\begin{gathered}
F:[0, T] \times \Omega \times \widetilde{X}_{a} \rightarrow X, \quad G(t): \Omega \times \widetilde{X}_{a} \rightarrow Y^{t}, \\
B(t): \Omega \times \widetilde{X}_{a} \rightarrow \gamma\left(H, X_{-1}^{t}\right), \quad C(t): \Omega \times \widetilde{X}_{a} \rightarrow \gamma\left(H_{2}, Y^{t}\right),
\end{gathered}
$$

for each $t \in[0, T]$, where $Y^{t}$ are Banach spaces. We put $G(t)(\omega, x)=G(t, \omega, x)$, $B(t)(\omega, x)=B(t, \omega, x)$ and $C(t)(\omega, x)=C(t, \omega, x)$ for $(t, \omega, x) \in[0, T] \times \Omega \times X$. Assuming (H1) and $a \in\left[0, \eta_{0}\right)$, we state our main hypotheses on $F, G, B$ and $C$.

(H2) For all $x \in \widetilde{X}_{a}$, the map $(t, \omega) \mapsto F(t, \omega, x)$ is strongly measurable and adapted. The function $F$ has linear growth and is Lipschitz continuous in space uniformly in $[0, T] \times \Omega$; that is, there are constants $L_{F}$ and $C_{F}$ such that for all $t \in[0, T], \omega \in \Omega$ and $x, y \in \widetilde{X}_{a}$ we have

$$
\begin{aligned}
\|F(t, \omega, x)-F(t, \omega, y)\|_{X} & \leq L_{F}\|x-y\|_{\widetilde{X}_{a}}, \\
\|F(t, \omega, x)\|_{X} & \leq C_{F}\left(1+\|x\|_{\widetilde{X}_{a}}\right) .
\end{aligned}
$$

(H3) For all $x \in \widetilde{X}_{a}$, the map $(t, \omega) \mapsto\left(-A_{w}(t)\right)^{-\theta_{G}} \Lambda_{G}(t) G(t, \omega, x) \in X$ is strongly measurable and adapted. The function $\left(-A_{w}\right)^{-\theta_{G}} \Lambda_{G} G$ has linear growth and is Lipschitz continuous in space uniformly in $[0, T] \times \Omega$; i.e., there are constants $L_{G}$ and $C_{G}$ such that for all $t \in[0, T], \omega \in \Omega$ and $x, y \in \widetilde{X}_{a}$ we have

$$
\begin{aligned}
\left\|\left(-A_{w}(t)\right)^{-\theta_{G}} \Lambda_{G}(t)(G(t, \omega, x)-G(t, \omega, y))\right\|_{X} & \leq L_{G}\|x-y\|_{\widetilde{X}_{a}}, \\
\left\|\left(-A_{w}(t)\right)^{-\theta_{G}} \Lambda_{G}(t) G(t, \omega, x)\right\|_{X} & \leq C_{G}\left(1+\|x\|_{\widetilde{X}_{a}}\right) .
\end{aligned}
$$

(H4) Let $\theta_{B} \in[0, \mu)$ satisfy $a+\theta_{B}<\frac{1}{2}$. For all $x \in \widetilde{X}_{a}$, the map $(t, \omega) \mapsto$ $\left(-A_{w}(t)\right)^{-\theta} B(t, \omega, x) \in \gamma\left(H_{1}, X\right)$ is strongly measurable and adapted. The function $\left(-A_{w}\right)^{-\theta_{B}} B$ has linear growth and is Lipschitz continuous in space uniformly in $[0, T] \times \Omega$; that is, there are constants $L_{B}$ and $C_{B}$ such that for all $t \in[0, T], \omega \in \Omega$ and $x, y \in \widetilde{X}_{a}$ we have

$$
\begin{aligned}
\left\|\left(-A_{w}(t)\right)^{-\theta_{B}}(B(t, \omega, x)-B(t, \omega, y))\right\|_{\gamma\left(H_{1}, X\right)} & \leq L_{B}\|x-y\|_{\widetilde{X}_{a}}, \\
\left\|\left(-A_{w}(t)\right)^{-\theta_{B}} B(t, \omega, x)\right\|_{\gamma\left(H_{1}, X\right)} & \leq C_{B}\left(1+\|x\|_{\widetilde{X}_{a}}\right) .
\end{aligned}
$$

(H5) Let $\theta_{C} \in[0, \mu)$ satisfy $a+\theta_{C}<\frac{1}{2}$. For all $x \in \widetilde{X}_{a}$, the mapping $(t, \omega) \mapsto\left(-A_{w}(t)\right)^{-\theta_{C}} \Lambda_{C}(t) C(t, \omega, x) \in \gamma\left(H_{2}, X\right)$ is strongly measurable and adapted. The function $\left(-A_{w}\right)^{-\theta_{C}} \Lambda_{C} C$ has linear growth and is Lipschitz continuous in space uniformly in $[0, T] \times \Omega$; that is, there are constants $L_{G}$ and $C_{G}$ such that for all $t \in[0, T], \omega \in \Omega$ and $x, y \in \widetilde{X}_{a}$ we have

$$
\begin{aligned}
\left\|\left(-A_{w}(t)\right)^{-\theta_{C}} \Lambda_{C}(t)(C(t, \omega, x)-C(t, \omega, y))\right\|_{\gamma\left(H_{2}, X\right)} & \leq L_{C}\|x-y\|_{\widetilde{X}_{a}}, \\
\left\|\left(-A_{w}(t)\right)^{-\theta_{C}} \Lambda_{C}(t) C(t, \omega, x)\right\|_{\gamma\left(H_{2}, X\right)} & \leq C_{C}\left(1+\|x\|_{\widetilde{X}_{a}}\right) .
\end{aligned}
$$

We introduce our first solution concept. 
Definition 3.1. Assume that (H1)-(H5) hold for some $\theta_{G}, \theta_{B}, \theta_{C} \geq 0$ and $a \in$ $\left[0, \eta_{0}\right)$. Let $r \in(2, \infty)$ satisfy $\min \left\{1-\theta_{G}, \frac{1}{2}-\theta_{B}, \frac{1}{2}-\theta_{C}\right\}>\frac{1}{r}$. We call an $\widetilde{X}_{a}{ }^{-}$ valued process $(U(t))_{t \in[0, T]}$ a mild solution of $(\mathrm{SE})$ if

(i) $U:[0, T] \times \Omega \rightarrow \widetilde{X}_{a}$ is strongly measurable and adapted, and we have $U \in L^{r}\left(0, T ; \widetilde{X}_{a}\right)$ almost surely,

(ii) for all $t \in[0, T]$, we have

$U(t)=P(t, 0) u_{0}+P * F(\cdot, U)(t)+P * \Lambda_{G} G(\cdot, U)(t)+P \diamond_{1} B(\cdot, U)(t)+P \diamond_{2} \Lambda_{C} C(\cdot, U)(t)$

in $X$ almost surely.

Here we have used the abbreviations

$$
P * \phi(t)=\int_{0}^{t} P(t, s) \phi(s) d s, \quad P \diamond_{k} \Phi(t)=\int_{0}^{t} P(t, s) \Phi(s) d W_{H_{k}}(s), \quad k=1,2,
$$

whenever the integrals are well-defined. Under our hypotheses both $P * F(\cdot, U)(t)$ and $P * \Lambda_{G} G(\cdot, U)(t)$ are in fact well-defined in $X$. Indeed, for the first one this is clear from (H2). For the second one we may write

$$
P(t, s) \Lambda_{G}(s) G(s, U(s))=P(t, s)\left(-A_{w}(s)\right)^{\theta_{G}}\left(-A_{w}(s)\right)^{-\theta_{G}} \Lambda_{G}(s) G(s, U(s))
$$

It then follows from (2.4), Hölder's inequality, and (H3) that

$$
\begin{aligned}
\int_{0}^{t} \| P(t, s) & \Lambda_{G}(s) G(s, U(s)) \|_{X} d s \\
& \lesssim \int_{0}^{t}(t-s)^{-\theta_{G}}\left\|\left(-A_{w}(s)\right)^{-\theta_{G}} \Lambda_{G}(s) G(s, U(s))\right\|_{X} d s \\
& \lesssim 1+\|U\|_{L^{r}\left(0, T ; \tilde{X}_{a}\right)},
\end{aligned}
$$

using that $1-\theta_{G}>\frac{1}{r}$. Similarly one can show that $P \diamond_{1} B(\cdot, U)(t)$ and $P \diamond_{2}$ $\Lambda_{C} C(\cdot, U)(t)$ are well-defined in $X$, taking into account Proposition 2.3: Estimate (2.4), Hölder's inequality and (H4) imply that

$$
\begin{aligned}
\int_{0}^{t} \| P(t, s) & B(s, U(s)) \|_{\gamma\left(H_{1}, X\right)}^{2} d s \\
& \lesssim \int_{0}^{t}(t-s)^{-2 \theta_{B}}\left\|\left(-A_{w}(s)\right)^{-\theta_{B}} B(s, U(s))\right\|_{\gamma\left(H_{1}, X\right)}^{2} d s \\
& \lesssim 1+\|U\|_{L^{r}\left(0, T ; \tilde{X}_{a}\right)}^{2}
\end{aligned}
$$

since $\frac{1}{2}-\theta_{B}>\frac{1}{r}$. In the same way it can be proved that the integral with respect to $W_{H_{2}}$ is well-defined.

We also recall the definition of a variational solution from [29]. To that purpose, for $t \in[0, T]$, we set

$$
\begin{aligned}
\Gamma_{t}=\left\{\varphi \in C^{1}\left([0, t] ; X^{*}\right)\right. & : \varphi(s) \in D\left(A(s)^{*}\right) \text { for all } s \in[0, t] \\
& \text { and } \left.\left[s \mapsto A(s)^{*} \varphi(s)\right] \in C\left([0, t] ; X^{*}\right)\right\} .
\end{aligned}
$$

Definition 3.2. Assume that (H1)-(H5) hold with $a \in\left[0, \eta_{0}\right)$. An $\widetilde{X}_{a}$-valued process $(U(t))_{t \in[0, T]}$ is called a variational solution of $(\mathrm{SE})$ if

(i) $U$ belongs to $L^{2}\left(0, T ; \widetilde{X}_{a}\right)$ a.s. and $U$ is strongly measurable and adapted,

(ii) for all $t \in[0, T]$ and all $\varphi \in \Gamma_{t}$, almost surely we have

$$
\begin{aligned}
\langle U(t), \varphi(t)\rangle-\left\langle u_{0}, \varphi(0)\right\rangle= & \int_{0}^{t}\left[\left\langle U(s), \varphi^{\prime}(s)\right\rangle+\left\langle U(s), A(s)^{*} \varphi(s)\right\rangle\right. \\
& \left.+\langle F(s, U(s)), \varphi(s)\rangle+\left\langle\Lambda_{G}(s) G(s, U(s)), \varphi(s)\right\rangle\right] d s \\
& +\int_{0}^{t} B(s, U(s))^{*} \varphi(s) d W_{H_{1}}(s)
\end{aligned}
$$




$$
+\int_{0}^{t}\left(\Lambda_{C}(s) C(s, U(s))\right)^{*} \varphi(s) d W_{H_{2}}(s) .
$$

The integrand $B(s, U(s))^{*} \varphi(s)$ in (3.2) should be read as

$$
\left(\left(-A_{w}(s)\right)^{-\theta_{B}} B(s, U(s))\right)^{*}\left(-A_{w}(s)^{*}\right)^{\theta_{B}} \varphi(s) .
$$

It follows from (H4) that the function $s \mapsto\left(\left(-A_{w}(s)\right)^{-\theta_{B}} B(s, U(s))\right)^{*}$ is $X^{*}$-strongly measurable. Moreover, the map

$$
s \mapsto\left(-A_{w}(s)^{*}\right)^{\theta_{B}} \varphi(s)=\left(-A_{w}(s)^{*}\right)^{-1+\theta_{B}}\left(-A_{w}(s)^{*}\right) \varphi(s)
$$

belongs to $C\left([0, t] ; X^{*}\right)$ by the Hölder continuity of $s \mapsto\left(-A_{w}(s)\right)^{-1+\theta_{B}}$ (cf. [25, (2.10) and (2.11)]) and the assumption on $\varphi$. Using (H4), we thus obtain that the integrand is contained in $L^{2}\left(0, T ; H_{1}\right)$ a.s.. As a result, the first stochastic integral in (3.2) is well-defined. The other integrands have to be interpreted similarly.

The next result shows that both solution concepts are equivalent in our setting. It follows from Proposition 5.4 and Remark 5.3 in [29] in the same way as Theorem 3.4 below. (Remark 5.3 can be used since $X$ is reflexive as a UMD space.)

Proposition 3.3. Assume that (H1)-(H5) hold for some $\theta_{G}, \theta_{B}, \theta_{C} \geq 0$ and $a \in$ $\left[0, \eta_{0}\right)$. Let $r \in(2, \infty)$ satisfy $\max \left\{\theta_{C}, \theta_{B}\right\}<\frac{1}{2}-\frac{1}{r}$ and $\theta_{G}<1-\frac{1}{r}$. Let $U$ : $[0, T] \times \Omega \rightarrow \widetilde{X}_{a}$ be a strongly measurable and adapted process such that $U$ belongs to $L^{r}\left(0, T ; \widetilde{X}_{a}\right)$ a.s.. Then $U$ is a mild solution of (SE) if and only if $U$ is a variational solution of $(\mathrm{SE})$.

We can now state the main existence and regularity result for (SE).

Theorem 3.4. Assume that (H1)-(H5) hold for some $\theta_{G}, \theta_{B}, \theta_{C} \geq 0$ and $a \in$ $\left[0, \eta_{0}\right)$. Let $u_{0}: \Omega \rightarrow \widetilde{X}_{a}^{0}$ be strongly $\mathcal{F}_{0}$ measurable. Then the following assertions hold.

(1) There is a unique mild solution $U$ of (SE) with paths in $C\left([0, T] ; \widetilde{X}_{a}\right)$ a.s..

(2) For every $\delta, \lambda>0$ with

$$
\delta+a+\lambda<\min \left\{1-\theta_{G}, \frac{1}{2}-\theta_{B}, \frac{1}{2}-\theta_{C}, \eta_{0}\right\}
$$

there exists a version of $U$ such that $U-P(\cdot, 0) u_{0}$ in $C^{\lambda}\left([0, T] ; \widetilde{X}_{\delta+a}\right)$ a.s..

(3) If $\delta, \lambda>0$ are as in (2) and if $u_{0} \in \widetilde{X}_{a+\delta+\lambda}$ a.s., then $U$ has a version with paths in $C^{\lambda}\left([0, T] ; \widetilde{X}_{\delta+a}\right)$ a.s.

Proof. Assertions (1) and (2) can be reduced to the case

$$
\left\{\begin{aligned}
d U(t) & =\left(A(t) U(t)+\tilde{F}(t, U(t))+\tilde{B}(t, U(t)) d W_{H}(t), t \in[0, T],\right. \\
U(0) & =u_{0} .
\end{aligned}\right.
$$

taking $\tilde{F}=F+\Lambda_{G} G$ and $\tilde{B}=\left(B, \Lambda_{C} C\right)$ and $H=H_{1} \times H_{2}$. The theorem now follows from [29, Theorem 6.3]. In view of (2), for assertion (3) we only have to show that $P(\cdot, 0) u_{0}$ has the required regularity, which is proved in [29, Lemma 2.3]. We note that, in order to apply the above results from [29] here, one has to replace in [29] the real interpolation spaces of type $(\eta, 2)$ by complex interpolation spaces of exponent $\eta$. This can be done using the arguments given in [29].

\section{BOUNDARY NOISE}

Let $S \subseteq \mathbb{R}^{d}$ be a bounded domain with $C^{2}$-boundary and outer unit normal vector of $n(s)$. On $S$ we consider the stochastic equation with boundary noise

$$
\begin{gathered}
\frac{\partial u}{\partial t}(t, s)=\mathcal{A}(t, s, D) u(t, s)+f(t, s, u(t, s)) \\
+b(t, s, u(t, s)) \frac{\partial w_{1}}{\partial t}(t, s),
\end{gathered}
$$

$$
s \in S, t \in(0, T],
$$




$$
\begin{array}{rlrl}
\mathcal{B}(t, s, D) u(t, s) & =G(t, u(t, \cdot))(s)+\tilde{C}(t, u(t, \cdot))(s) \frac{\partial w_{2}}{\partial t}(t, s), & & s \in \partial S, t \in(0, T], \\
u(0, s) & =u_{0}(s), & s \in S .
\end{array}
$$

Here $w_{k}$ are Brownian motions as specified below, and we use the differential operators

$$
\mathcal{A}(t, s, D)=\sum_{i, j=1}^{d} D_{i}\left(a_{i j}(t, s) D_{j}\right)+a_{0}(t, s), \quad \mathcal{B}(t, s, D)=\sum_{i, j=1}^{d} a_{i j}(t, s) n_{i}(s) D_{j} .
$$

For simplicity we only consider the case of a scalar equation, but systems could be treated in the same way, cf. e.g. [5, 15].

(A1) We assume that the coefficients of $\mathcal{A}$ and $\mathcal{B}$ are real and satisfy

$$
\begin{aligned}
a_{i j} & \in C^{\mu}([0, T] ; C(\bar{S})), a_{i j}(t, \cdot) \in C^{1}(\bar{S}), D_{k} a_{i j} \in C([0, T] \times \bar{S}), \\
a_{0} & \in C^{\mu}\left([0, T], L^{d}(S)\right) \cap C([0, T] ; C(\bar{S}))
\end{aligned}
$$

for a constant $\mu \in\left(\frac{1}{2}, 1\right]$ and all $i, j, k=1, \ldots, d$ and $t \in[0, T]$. Further, let $\left(a_{i j}\right)$ be symmetric and assume that there is a $\kappa>0$ such that

$$
\sum_{i, j=1}^{d} a_{i j}(t, s) \xi_{i} \xi_{j} \geq \kappa|\xi|^{2} \quad \text { for all } s \in \bar{S}, t \in[0, T], \xi \in \mathbb{R}^{d} .
$$

In the following we reformulate the problem (4.1) as (SE) thereby giving (4.1) a precise sense. Set $X=L^{p}(S)$ for some $p \in(1, \infty)$. Let $\alpha \in[0,2]$ satisfy $\alpha-\frac{1}{p} \neq 1$. We introduce the space

$$
H_{\mathcal{B}(t)}^{2, p}(S)= \begin{cases}\left\{f \in H^{\alpha, p}(S): \mathcal{B}(t, \cdot, D) f=0\right\}, & \alpha-\frac{1}{p}>1, \\ H^{\alpha, p}(S), & \alpha-\frac{1}{p}<1,\end{cases}
$$

where $H^{\alpha, p}(S)$ denotes the usual Bessel-potential space (see [28]). We also set

$$
\widetilde{X}_{\eta}=H^{2 \eta, p}(S) \quad \text { for all } \eta \geq 0 .
$$

We further define $A(t): D(A(t)) \rightarrow X$ by $A(t) x=\mathcal{A}(t, \cdot, D) x$ and

$$
D(A(t))=\left\{x \in H^{2, p}(S): \mathcal{B}(t, \cdot, D) x=0\right\}=H_{\mathcal{B}(t)}^{2, p}(S) .
$$

Lemma 4.1. Let $X=L^{p}(S)$ and $p \in(1, \infty)$. Assume that $(A 1)$ is satisfied. The following assertions hold.

(1) The operators $A(t), t \in[0, T]$, satisfy (AT) and the graph norms of $A(t)$ are uniformly equivalent with $\|\cdot\|_{H^{2, p}(S)}$. In particular, $(A(t))_{t \in[0, T]}$ generates a unique strongly continuous evolution family $(P(t, s))_{0 \leq s \leq t \leq T}$ on $X$.

(2) We have $X_{\theta}^{t}=H_{\mathcal{B}(t)}^{2 \theta, p}(S)$ for all $\theta \in(0,1)$ with $2 \theta-\frac{1}{p} \neq 1$, as well as $X_{\eta}^{t}=\widetilde{X}_{\eta}=H^{2 \eta, p}(S)$ for all $\eta \in\left[0, \frac{1}{2}+\frac{1}{2 p}\right)$, in the sense of isomorphic Banach spaces. The norms of these isomorphisms are bounded uniformly for $t \in[0, T]$.

(3) Let $p \in[2, \infty)$. Then condition (H1) holds with $\eta_{0}=1 / 2$.

Proof. (1): See [1] and [30]. Note that $A(t)^{*}$ on $L^{p^{\prime}}(S)=X^{*}$ is given by $A^{*}(t) \varphi=$ $\mathcal{A}(t, \cdot, D) \varphi$ with $D\left(A(t)^{*}\right)=H_{\mathcal{B}(t)}^{2, p^{\prime}}(S)$, and thus also $\left(A(t)^{*}\right)_{0 \leq t \leq T}$ satisfies (AT).

(2): Let $\theta \in(0,1)$ and $p \in(1, \infty)$ satisfy $2 \theta-\frac{1}{p} \neq 1$. Then Theorem 5.2 and Remark 5.3(c) in [5] show that

$$
X_{\theta}^{t}=\left[L^{p}(S), D(A(t))\right]_{\theta}=\left[L^{p}(S), H_{\mathcal{B}(t)}^{2, p}(S)\right]_{\theta}=H_{\mathcal{B}(t)}^{2 \theta, p}(S)
$$

isomorphically, see also [26, Theorem 4.1] and [28, Theorem 1.15.3]. Inspecting the proofs given in [26] one sees that the isomorphisms in (4.3) are bounded uniformly in $t \in[0, T]$. Similarly, if $2 \theta-\frac{1}{p}<1$, then $X_{\theta}^{t}=H_{\mathcal{B}(t)}^{2 \theta, p}(S)=H^{2 \theta, p}(S)=\widetilde{X}_{\theta}$. 
(3): This is clear from (1), (2) and the definitions. Note that the spaces $\widetilde{X}_{\eta}^{t}$ are UMD spaces with type 2 because they are isomorphic to closed subspaces of $L^{p}$-spaces with $p \in[2, \infty)$.

Remark 4.2. Let the constant $w \geq 0$ be given by (AT). In problem (4.1) we replace $\mathcal{A}$ and $f$ by $\mathcal{A}-w$ and $f+w$, respectively, without changing the notation. This modification does not affect the assumptions (A1) and (A2), and from now we can thus take $w=0$ in (AT).

Next, we apply Theorem 9.2 and Remark 9.3(e) of [5] in order to construct the operators $\Lambda_{C}(t)$ and $\Lambda_{D}(t)$. In [5] it is assumed that $\partial S \in C^{\infty}$. However, the results from [5] used below remain valid under our assumption that $\partial S \in C^{2}$, due to Remark 7.3 of [5] combined with Theorem 2.3 of [14].

Let $t \in[0, T]$. In view of our main Theorem 4.3 we consider only $p \geq 2$ and $\alpha \in\left(1,1+\frac{1}{p}\right)$ though some of the results stated below can be generalized to other exponents. Let

$$
Y=\partial W^{\alpha, p}(S):=W^{\alpha-1-1 / p, p}(\partial S)
$$

be the Slobodeckii space of negative order on the boundary which is defined via duality e.g. in (5.16) of [5]. Let $y \in Y$. Theorem 9.2 and Remark 9.3(e) of [5] give a unique weak solution $x \in H^{\alpha, p}(S)$ of the elliptic problem

$$
\begin{array}{ll}
\mathcal{A}(t, \cdot, D) x=0 & \text { on } S, \\
\mathcal{B}(t, \cdot, D) x=y & \text { on } \partial S .
\end{array}
$$

(Weak solutions are defined by means of test functions $v \in H^{2-\alpha, p^{\prime}}(S)$, see $[5$, (9.4)].) We set $N(t) y:=x$. Formula (9.15) of [5] implies that the 'Neumann map' $N(t)$ belongs $\mathcal{B}\left(\partial W^{\alpha, p}(S), H^{\alpha, p}(S)\right)$ and that the map $N(\cdot):[0, T] \rightarrow$ $\mathcal{B}\left(\partial W^{\alpha, p}(S), H^{\alpha, p}(S)\right)$ is continuous.

Concerning the other terms in the first line of (4.1) and the noise terms, we make the following hypotheses.

(A2) The functions $f, b:[0, T] \times \Omega \times S \times \mathbb{R} \rightarrow \mathbb{R}$ are jointly measurable, adapted to $\left(\mathcal{F}_{t}\right)_{t \geq 0}$, and Lipschitz functions and of linear growth in the third variable, uniformly in the other variables.

(A3) For $k=1,2$, the process $w_{k}$ can be written in the form $i_{k} W_{H_{k}}$, where $i_{1} \in \gamma\left(H_{1}, L^{r}(S)\right)$ for some $r \in[1, \infty)$ and $i_{2} \in \gamma\left(H_{2}, L^{s}(\partial S)\right)$ for some $s \in[1, \infty)$, and $W_{H_{1}}$ and $W_{H_{2}}$ are independent $H_{k}$-cylindrical Brownian motions with respect to $\left(\mathcal{F}_{t}\right)_{t \geq 0}$.

Supposing that (A2) holds, we define $F:[0, T] \times \Omega \times X \rightarrow X$ by setting $F(t, \omega, x)(s)=f(t, \omega, s, x(s))$. Then $F$ satisfies (H2). We further define the function $B(t, \omega, x) h$ on $S$ for $(t, \omega, x) \in[0, T] \times \Omega \times X$ and $h \in H_{1}$ by means of

$$
B(t, \omega, x) h=b(t, \omega, \cdot, x(\cdot)) i_{1} h
$$

In Examples 4.7 and 4.8 we give conditions on $w_{1}$ and $\theta_{B}$ such that $(-A)^{-\theta_{B}} B$ maps $[0, T] \times \Omega \times X$ into $\gamma\left(H_{1}, X\right)$ and (H4) holds.

Assumption (A3) has to be interpreted in the sense that

$$
w_{k}(t, s)=\sum_{n \geq 1}\left(i_{k} h_{n}^{k}\right)(s) W_{H_{k}}(t) h_{n}^{k}, \quad t \in \mathbb{R}_{+}, s \in S, k=1,2,
$$

where $\left(h_{n}^{k}\right)_{n \geq 1}$ is an orthonormal basis for $H_{k}$, and the sum converges in $L^{r}(S)$ if $k=1$ and in $L^{s}(\partial S)$ if $k=2$. We note that then $\left(w_{k}(t, \cdot)\right)_{t>0}$ is a Brownian motion with values in $L^{r}(S)$ and $L^{s}(\partial S)$, respectively. Conversely, if $\left(w_{k}(t, \cdot)\right)_{t \geq 0}, k=1,2$, are independent Brownian motions with values in $L^{r}(S)$ and $L^{s}(\partial S)$, then we can always construct $i_{k}$ and $W_{H_{k}}$ as above, cf. Example 4.6 below. 
We recall that $H^{\alpha, p}(S)=X_{\frac{\alpha}{2}}^{t}$ for $t \in[0, T]$ and $\alpha \in\left(1,1+\frac{1}{p}\right)$ by Lemma 4.1(2). Moreover, the operator $A(t)$ has bounded imaginary powers in $X$ (uniformly in $t \in[0, T]$ ), see e.g. Example 4.7.3(d) and Section 4.7 in [6]. It then follows that

$$
H^{\alpha, p}(S)=X_{\frac{\alpha}{2}}^{t}=D\left((-A(t))^{\frac{\alpha}{2}}\right)
$$

with uniformly equivalent norms for $t \in[0, T]$, see e.g. [28, Theorem 1.15.3]. Therefore, the extrapolated operator $A_{-1}(t)$ maps $H^{\alpha, p}(S)$ into $X_{\frac{\alpha}{2}-1}^{t}$, and hence

$$
\Lambda(t)=\Lambda_{G}(t)=\Lambda_{C}(t):=-A_{-1}(t) N(t) \in \mathcal{B}\left(Y, X_{\frac{\alpha}{2}-1}^{t}\right)
$$

with uniformly bounded norms for $t \in[0, T]$. Let $\theta \in\left[1-\frac{\alpha}{2}, 1\right]$. As above, we further obtain $X_{\frac{\alpha}{2}-1+\theta}^{t}=H^{\alpha-2+2 \theta, p}(S) \hookrightarrow X$, so that

$$
(-A(t))^{-\theta} \Lambda(t) \in \mathcal{B}\left(Y, H^{\alpha-2+2 \theta, p}(S)\right)
$$

with uniformly bounded norms for $t \in[0, T]$.

In order to relate the boundary noise term in (4.1) with (SE), we set

$$
(C(t, \omega, x) h)(s)=\tilde{C}(t, \omega, x)(s)\left(i_{2} h\right)(s)
$$

for $h \in H_{2}$. We aim at the mapping property $C(t, \omega, x): H_{2} \rightarrow Y=\partial W^{\alpha, p}(S)$ since it will enable us to verify the hypothesis (H5). In fact, if $C(t, \omega, x) \in \mathcal{B}\left(H_{2}, Y\right)$ then $(-A(t))^{-\theta} \Lambda(t) C(t, \omega, x)$ maps $H_{2}$ continuously into $H^{\alpha-2+2 \theta, p}(S) \hookrightarrow X$ if $\theta \in\left[1-\frac{\alpha}{2}, 1\right]$. In Examples 4.4 and 4.6 we give conditions on $\tilde{C}$ and $i_{2}$ implying (H5) for $\stackrel{C}{C}$. The deterministic boundary term $G$ can be treated in a similar way.

We want to present a variational formulation of (4.1), starting with an informal discussion. Let $\varphi \in \Gamma_{t}$, where $\Gamma_{t}$ is given by (3.1). Then $\varphi(r) \in D\left(A(r)^{*}\right)=$ $W_{\mathcal{B}(r)}^{2, p^{\prime}}(S)$. Formally, multiplying (4.1) by $\varphi$, integrating over $[0, t] \times S$, integrating by parts and interchanging the order of integration, we obtain that, almost surely,

$$
\begin{aligned}
\int_{S}\left[u(t, s) \varphi(t)(s)-u_{0}(s) \varphi(0)(s)\right] d s & =\int_{0}^{t} \int_{S} u(r, s)\left[\mathcal{A}(r, \cdot, D) \varphi(r)+\varphi^{\prime}(r)\right](s) d s d r \\
& +\int_{0}^{t} \int_{S} f(r, s, u(r, s)) \varphi(r)(s) d s d r \\
& +\int_{S} \int_{0}^{t} b(r, s, u(r, s)) \varphi(r)(s) d w_{1}(r, s) d s+T_{1}
\end{aligned}
$$

In the boundary term $T_{1}$ the part with $\nabla \varphi(r)$ disappears since $\varphi(r) \in D\left(A(r)^{*}\right)$, and the other term is given by

$$
\begin{aligned}
T_{1} & =\int_{\partial S} \int_{0}^{t} \mathcal{B}(r, \cdot, D) u(r, \cdot) \operatorname{tr}(\varphi(r)) d r d \sigma \\
& =\int_{\partial S} \int_{0}^{t} G(r, u(r, \cdot)) \operatorname{tr}(\varphi(r)) d r d \sigma+\int_{\partial S} \int_{0}^{t} \tilde{C}(r, u(r, \cdot)) \operatorname{tr}(\varphi(r)) d w_{2}(r, \cdot) d \sigma
\end{aligned}
$$

where tr denotes the trace operator on $W_{\mathcal{B}(r)}^{2, p^{\prime}}(S)$.

We now start from the equation (4.7) and rewrite it using (2.7) and the notation introduced above. Setting $u(t, s)=: U(t)(s)$, equality (4.7) becomes

$$
\begin{aligned}
\langle U(t), \varphi(t)\rangle-\left\langle u_{0}, \varphi(0)\right\rangle= & \int_{0}^{t}\left\langle U(r),\left(\mathcal{A}(r, \cdot, D) \varphi(r)+\varphi^{\prime}(r)\right\rangle d r+T_{1}\right. \\
& +\int_{0}^{t}\langle F(r, U(r)), \varphi(r)\rangle d r+\int_{0}^{t} B(r, U(r))^{*} \varphi(r) d W_{H_{1}}(r),
\end{aligned}
$$

and the boundary term yields

$$
T_{1}=\int_{0}^{t}\langle G(r, U(r)), \operatorname{tr}(\varphi(r))\rangle d r+\int_{0}^{t} C(r, U(r))^{*} \operatorname{tr}(\varphi(r)) d W_{H_{2}}(r) .
$$


Here the brackets denote the duality pairing on $L^{p}(S)$ and $L^{p}(\partial S)$, respectively. We claim that for all $x \in W_{\mathcal{B}(t)}^{2, p^{\prime}}(S)$ it holds

$$
\operatorname{tr}(x)=\Lambda(t)^{*} x=\left(-A_{-1}(t) N(t)\right)^{*} x .
$$

Indeed, let $\alpha \in\left(1,1+\frac{1}{p}\right), x \in W_{\mathcal{B}(t)}^{2, p^{\prime}}(S)=D\left(A^{*}(t)\right)$ and $y \in Y=\partial W^{\alpha, p}(S)$. Then we have $N(t) y \in H^{\alpha, p}(S)$ and $a(t) \nabla x \cdot n=0$ on $\partial S$. Observe that $\Lambda(t)^{*}$ maps $D\left(A(t)^{*}\right)$ into $Y^{*}$. Integrating by parts and using formula (9.4) of [5], we obtain

$$
\begin{aligned}
\left\langle y, \Lambda(t)^{*} x\right\rangle_{Y} & =\langle\Lambda(t) y, x\rangle_{X_{-1}^{t}}=-\left\langle N(t) y, A(t)^{*} x\right\rangle_{X} \\
& =-\int_{S} N(t) y\left[\nabla \cdot(a(t) \nabla x)+a_{0}(t) x\right] d s \\
& =0+\int_{S}\left[(a(t) \nabla N(t) y) \cdot \nabla x+a_{0}(t)(N(t) y) x\right] d s=\langle y, \operatorname{tr}(x)\rangle_{Y},
\end{aligned}
$$

which proves the claim. Therefore, $T_{1}$ becomes

$$
T_{1}=\int_{0}^{t}\langle\Lambda(t) G(r, U(r)), \varphi(r)\rangle d r+\int_{0}^{t}(\Lambda(t) C(r, U(r)))^{*} \varphi(r) d W_{H_{2}}(r) .
$$

Combining this expression with (4.8) we arrive at the definition of a variational solution to the stochastic evolution equation (4.1), as introduced in Definition 3.2. The above calculations thus motivate the following definitions. We say $u$ is a variational (resp. mild) solution to (4.1) if $U(t)(s)=u(t, s)$ is a variational (resp. mild) solution to (SE) with the above definitions of $A(t), F, \Lambda_{G}, G, B, \Lambda_{C}, C$ and $W_{H_{k}}$. We can now state out main result.

Theorem 4.3. Let $p \in[2, \infty), X=L^{p}(S), \alpha \in\left(1,1+\frac{1}{p}\right), \theta_{B} \in\left[0, \frac{1}{2}\right), \theta_{C} \in$ $\left(1-\frac{\alpha}{2}, \frac{1}{2}\right)$ and $\theta_{G} \in\left(1-\frac{\alpha}{2}, 1\right)$. Assume that (A1)-(A3) and (H3)-(H5) hold, where $C, G, B, \Lambda_{C}$ and $\Lambda_{G}$ are defined above. Let $u_{0}: \Omega \rightarrow X$ be strongly $\mathcal{F}_{0}$-measurable. Then the following assertions are true.

(1) There exists a unique variational and mild solution $u$ of (4.1) with paths in $C([0, T] ; X)$ a.s..

(2) For every $\delta, \lambda>0$ with $\delta+\lambda<\min \left\{1-\theta_{G}, \frac{1}{2}-\theta_{B}, \frac{1}{2}-\theta_{C}\right\}$ there exists a version of $u$ such that $u-P(\cdot, 0) u_{0}$ in $C^{\lambda}\left([0, T] ; \tilde{X}_{\delta}\right)$ a.s..

(3) If $\delta, \lambda>0$ are as in (2) and if $u_{0} \in \widetilde{X}_{\delta+\lambda}$ a.s., then $u$ has a version with paths in $C^{\lambda}\left([0, T] ; \widetilde{X}_{\delta}\right)$.

Note that we need $\frac{1}{2}-\theta_{C}<\frac{\alpha}{2}-\frac{1}{2}<\frac{1}{2 p}$. Thus, if $\frac{1}{2}-\theta_{B} \geq \frac{1}{2 p}, 1-\theta_{G} \geq \frac{1}{2 p}$ and the other assumptions in Theorem 4.3 hold, then we can take $\lambda, \delta \geq 0$ with $\delta+\lambda<\frac{1}{2 p}$ and deduce that $u-P(\cdot, 0) u_{0}$ belongs to $C^{\lambda}\left([0, T] ; \widetilde{X}_{\delta}\right)$ a.s.. If we also have $u_{0} \in H^{\frac{1}{p}, p}(S)$, then we obtain a solution $u$ of $(4.1)$ with paths in $C\left([0, T] ; H^{2 \delta, p}(S)\right)$ for all $\delta<\frac{1}{2 p}$. In this case Sobolev's embedding (see [28, Theorem 4.6.1]) implies that

$$
u \in C\left([0, T] ; L^{q}(S)\right) \text { for all } \begin{cases}q<\frac{d p}{d-1} & \text { if } d \geq 2 \\ q<\infty, & \text { if } d=1\end{cases}
$$

Proof of Theorem 4.3. The existence and uniqueness of a mild solution with the asserted regularity follows from Theorem 3.4 and the above observations. The equivalence with the variational solution is a consequence of Proposition 3.3.

We now discuss several examples under which (H4) and (H5) hold. The hypothesis (H3) can be treated in the same way. We start with some observations concerning Gaussian random variables $\xi$ with values in a Banach space $Z$, see e.g. [7], [9] and the references therein. The covariance $Q \in \mathcal{B}\left(Z^{*}, Z\right)$ of $\xi$ is given by 
$Q x^{*}=\mathbb{E}\left(\left\langle\xi, x^{*}\right\rangle \xi\right)$ for $x^{*} \in Z^{*}$. One introduces an inner product $[\cdot, \cdot]$ on the range of $Q$ by setting

$$
\left[Q x^{*}, Q y^{*}\right]:=\left\langle Q x^{*}, y^{*}\right\rangle=\mathbb{E}\left(\left\langle\xi, x^{*}\right\rangle\left\langle\xi, y^{*}\right\rangle\right)
$$

for $x^{*}, y^{*} \in Z^{*}$, and we define $\left\|Q x^{*}\right\|_{H}^{2}=\left[Q x^{*}, Q x^{*}\right]$. The reproducing kernel Hilbert space $H$ of $\xi$ is the completion of $Q Z^{*}$ with respect to $\|\cdot\|_{H}$. Then the identity on $Q Z^{*}$ can be extended to a continuous embedding $i: H \hookrightarrow E$, and it holds $Q=i i^{*}$. On the other hand, the random variables $w_{k}(t, \cdot)$ in (A3) are Gaussian with covariance $Q_{k}=t i_{k} i_{k}^{*}$ for all $t \geq 0$ and $k=1,2$.

Example 4.4. Let (A3) hold with $H_{2}=L^{2}(\partial S)$. Assume that covariance operator $Q_{2} \in \mathcal{B}\left(L^{2}(\partial S)\right)$ of $w_{2}$ is compact. Then there exist numbers $\left(\lambda_{n}\right)_{n \geq 1}$ in $\mathbb{R}_{+}$and an orthonormal system $\left(e_{n}\right)_{n \geq 1}$ in $L^{2}(\partial S)$ such that

$$
Q_{2}=\sum_{n \geq 1} \lambda_{n} e_{n} \otimes e_{n}
$$

Assume that

$$
\sum_{n \geq 1} \lambda_{n}\left\|e_{n}\right\|_{\infty}^{2}<\infty
$$

We observe that the operator $i_{2}$ is given by $i_{2}=\sum_{n \geq 1} \sqrt{\lambda_{n}} e_{n} \otimes e_{n}$ and belongs to $\mathcal{B}\left(L^{2}(\partial S), L^{\infty}(\partial S)\right)$. Let $p \in[2, \infty)$. Assume that $\tilde{C}:[0, T] \times \Omega \times L^{p}(S) \rightarrow L^{p}(\partial S)$ is strongly measurable and adapted, as well as Lipschitz and of linear growth in the third variable uniformly in $[0, T] \times \Omega$. Then (H5) holds for $C=\tilde{C} i_{2}$ with $a=0$ and every $\theta_{C} \in\left(1-\frac{\alpha}{2}, \frac{1}{2}\right)$, where $\alpha \in\left(1,1+\frac{1}{p}\right)$.

Proof. Lemma 2.2 implies that $i_{2} \in \gamma\left(H_{2}, L^{p}(\partial S)\right)$. Fix $t \in[0, T], \omega \in \Omega$ and $x, y \in X=L^{p}(S)$. Denote $K=\left\|i_{2}\right\|_{\mathcal{B}\left(H_{2}, L^{\infty}(\partial S)\right)}$. The embedding $L^{p}(\partial S) \hookrightarrow Y=$ $\partial W^{\alpha, p}(S)$ and (2.5) yield

$$
\|C(t, \omega, x)-C(t, \omega, y)\|_{\gamma\left(H_{2}, Y\right)} \lesssim_{p, \alpha}\|C(t, \omega, x)-C(t, \omega, y)\|_{\gamma\left(H_{2}, L^{p}(\partial S)\right) .}
$$

Furthermore, for $h \in H_{2}$ and $s \in S$ we have

$$
\begin{aligned}
|((C(t, \omega, x)-C(t, \omega, y)) h)(s)| & =|\tilde{C}(t, \omega, x)(s)-\tilde{C}(t, \omega, y)(s)|\left|i_{2} h(s)\right| \\
& \leq K|\tilde{C}(t, \omega, x)(s)-\tilde{C}(t, \omega, y)(s)|\|h\|_{H_{2}} .
\end{aligned}
$$

Lemma 2.2 and the assumptions of the example then imply that

$$
\begin{aligned}
\|C(t, \omega, x)-C(t, \omega, y)\|_{\gamma\left(H_{2}, L^{p}(\partial S)\right)} & \lesssim p K\|\tilde{C}(t, \omega, x)-\tilde{C}(t, \omega, y)\|_{L^{p}(\partial S)} \\
& \leq K L_{\tilde{C}}\|x-y\|_{L^{p}(S)} .
\end{aligned}
$$

Using (4.6), we can now deduce the first part of (H5). The second part is shown in a similar way.

Remark 4.5. Note that in Example 4.4 the noise could be a bit more irregular since in (4.10) one can still regain some integrability by choosing $\alpha$ and $\theta_{C}$ appropriately.

Example 4.6. Let $q \in(p, \infty]$ and $s \in[p, \infty)$ satisfy $\frac{1}{p}=\frac{1}{q}+\frac{1}{s}$. We assume that $w_{2}$ is an $L^{s}(\partial S)$-valued Brownian motion. Let $H_{2}$ be the reproducing kernel Hilbert space of the Gaussian random variable $w_{2}(1)=w_{2}(1, \cdot)$ with covariance $Q$ and $i_{2}$ be the embedding of $H_{2}$ into $L^{s}(\partial S)$. Then we have $i_{2} \in \gamma\left(H_{2}, L^{s}(\partial S)\right.$ ) (cf. [7], [9] and the references therein for details). It is easy to check that $t^{-1 / 2} w_{2}(t)$ also has the covariance $Q$ for $t>0$. Due to Proposition 2.6.1 in [17] we thus obtain

$$
t^{-1 / 2} w_{2}(t)=\sum_{n \geq 1}\left\langle t^{-1 / 2} w_{2}(t), x_{n}^{*}\right\rangle Q x_{n}^{*}
$$

in $X$ a.s. for every orthonormal basis $\left(Q x_{n}^{*}\right)_{n \geq 1}$ of $H_{2}$. Therefore

$$
w_{2}(t)=\sum_{n \geq 1}\left\langle w_{2}(t), x_{n}^{*}\right\rangle Q x_{n}^{*}
$$


converges in $X$ a.s.. We now define $W_{H_{2}}(t): Q L^{s^{\prime}}(\partial S) \rightarrow L^{2}(\Omega)$ by setting

$$
W_{H_{2}}(t) Q x^{*}=\sum_{n \geq 1}\left\langle w_{2}(t), x_{n}^{*}\right\rangle\left\langle Q x_{n}^{*}, x^{*}\right\rangle=\left\langle w_{2}(t), x^{*}\right\rangle
$$

for each $x^{*} \in L^{s^{\prime}}(\partial S)$ and $t \geq 0$. Then we deduce $\left\|W_{H_{2}}(t) Q x^{*}\right\|_{2}^{2}=\left\langle Q x^{*}, x^{*}\right\rangle=$ $\left\|Q x^{*}\right\|_{H_{2}}^{2}$ from (4.9), and thus $W_{H_{2}}$ extends to a bounded operator from $H_{2}$ into $L^{2}(\Omega)$. It is easy to check that $W_{H_{2}}$ is the required cylindrical Brownian motion with $w_{2}=i_{2} W_{H_{2}}$; i.e., (A3) holds for $k=2$. Assume that $\tilde{C}:[0, T] \times \Omega \times X: \rightarrow L^{q}(\partial S)$ is strongly measurable and adapted, as well as Lipschitz and of linear growth in the third variable uniformly in $[0, T] \times \Omega$. Then (H5) holds for $C=\tilde{C} i_{2}$, where we take $a=0, \theta_{C} \in\left(1-\frac{\alpha}{2}, \frac{1}{2}\right)$ and $\alpha \in\left(1,1+\frac{1}{p}\right)$.

Proof. Fix $t \in[0, T], \omega \in \Omega$ and $x, y \in X=L^{p}(S)$. We argue as in the previous example, but in (4.11) we consider $\tilde{C}(t, \omega, x)-\tilde{C}(t, \omega, y)$ as an multiplication operator from $L^{s}(\partial S)$ to $L^{p}(\partial S)$. Using Hölder's inequality and (2.5), we thus obtain

$$
\begin{aligned}
\|C(t, \omega, x)-C(t, \omega, y)\|_{\gamma\left(H_{2}, Y\right)} & \lesssim_{p, \alpha}\|C(t, \omega, x)-C(t, \omega, y)\|_{\gamma\left(H_{2}, L^{p}(\partial S)\right)} \\
& \leq\|\tilde{C}(t, \omega, x)-\tilde{C}(t, \omega, y)\|_{L^{q}(\partial S)}\left\|i_{2}\right\|_{\gamma\left(H_{2}, L^{s}(\partial S)\right)} \\
& \leq L_{\tilde{C}}\|x-y\|_{L^{p}(\partial S)}\left\|i_{2}\right\|_{\gamma\left(H_{2}, L^{s}(\partial S)\right)} .
\end{aligned}
$$

The first part of (H5) now follows in view of (4.6). The second part can be proved in the same way.

We now come to condition (H4).

Example 4.7. Assume that (A1)-(A3) hold with $r \in(d, \infty)$. Then $(\mathrm{H} 4)$ is satisfied for all $\theta_{B} \in\left(\frac{d}{2 r}, \frac{1}{2}\right)$.

Proof. Let $\frac{1}{q}=\frac{1}{p}+\frac{1}{r}$ and $\theta_{B} \in\left(\frac{d}{2 r}, \frac{1}{2}\right)$. As in Example 5.5 of [24] one can show

$$
L^{q}(S) \hookrightarrow X_{-\theta_{B}}^{t},
$$

where the embedding is uniformly bounded for $t \in[0, T]$. Fix $t \in[0, T], \omega \in \Omega$ and $x, y \in X=L^{p}(S)$. Arguing as in the previous example, by means of (4.12), (4.4), Hölder's inequality, (A2) and (2.5) we can estimate

$$
\begin{aligned}
\|(-A(t))^{-\theta_{B}} & (B(t, \omega, x)-B(t, \omega, y)) \|_{\gamma\left(H_{1}, X\right)} \\
& \lesssim \theta_{B}, p, r, n\|B(t, \omega, x)-B(t, \omega, y)\|_{\left.\gamma\left(H_{1}, L^{q}(S)\right)\right)} \\
& \leq\|b(t, \omega, x)-b(t, \omega, y)\|_{L^{p}(S)}\left\|i_{1}\right\|_{\gamma\left(H_{2}, L^{r}(S)\right)} \\
& \leq L_{b}\|x-y\|_{L^{p}(S)}\left\|i_{1}\right\|_{\gamma\left(H_{2}, L^{r}(S)\right)} .
\end{aligned}
$$

This proves the first part of (H4). The second part is obtained in a similar way.

Finally, we consider the white noise situation in the case $d=1$.

Example 4.8. Let $d=1$ and $p>2$ and assume that (A1)-(A3) hold with $i_{1}=I$. Then (H4) is satisfied for all $\theta_{B} \in\left(\frac{1}{2 p}+\frac{1}{4}, \frac{1}{2}\right)$.

Proof. Let $\frac{1}{q}=\frac{1}{p}+\frac{1}{2}$ and $\theta_{B} \in\left(\frac{1}{2 p}+\frac{1}{4}, \frac{1}{2}\right)$. Fix $t \in[0, T], \omega \in \Omega$ and $x, y \in X=$ $L^{p}(S)$. Observe that $(-A(t))^{-\theta_{B}}$ can be extended to $L^{q}(S)$ where it coincides with the fractional power of the corresponding realization $A_{q}(t)$ of $\mathcal{A}(t, \cdot, D)$ on $L^{q}(S)$ with the boundary condition $\mathcal{B}(t, \cdot, D) v=0$. We further obtain

$$
D\left(\left(-A_{q}(t)\right)^{\theta_{B}}\right) \hookrightarrow\left(L^{q}(S), H^{2, q}(S)\right)_{\theta_{B}, \infty} \hookrightarrow\left[L^{q}(S), H^{2, q}(S)\right]_{\vartheta}=H^{2 \vartheta, q}(S) .
$$

for $\vartheta \in\left(\frac{1}{2 p}+\frac{1}{4}, \theta_{B}\right)$ with uniform embedding constants, see Sections 1.10.3 and 1.15.2 of $[28]$ and (4.3). Sobolev's embedding then yields that $D\left(\left(-A_{q}(t)\right)^{\theta_{B}}\right) \hookrightarrow C(\bar{S})$. Using also Hölder's inequality, we thus obtain

$$
\left|\left[\left((-A(t))^{-\theta_{B}}(B(t, \omega, x)-B(t, \omega, y)) h\right)\right](s)\right| \lesssim \theta_{B}, p\|(B(t, \omega, x)-B(t, \omega, y)) h\|_{L^{q}(S)}
$$




$$
\begin{aligned}
& \leq\|b(t, \omega, x)-b(t, \omega, y)\|_{L^{p}(S)}\|h\|_{L^{2}(S)} \\
& \leq L_{b}\|x-y\|_{L^{p}(S)}\|h\|_{L^{2}(S)}
\end{aligned}
$$

for all $s \in S$. Now we can apply Lemma 2.2 to obtain that

$$
\left\|(-A(t))^{-\theta_{B}}(B(t, \omega, x)-B(t, \omega, y))\right\|_{\gamma\left(H_{1}, X\right)} \lesssim_{\theta_{B}, p, n} L_{b}\|x-y\|_{L^{p}(S)} .
$$

The other condition (H4) can be verified in the same way.

In the next remark we explain why one cannot consider Dirichlet boundary conditions with the above methods. This problem was not stated clearly in [20]. In the one dimensional case with $S=\mathbb{R}_{+}$, a version of (4.1) with Dirichlet boundary conditions has been treated in [4] using completely other methods and working on a weighted $L^{p}$ space on $\mathbb{R}_{+}$.

Remark 4.9. Since we are looking for a solution in $X=L^{p}(S)$, we have to require that $\alpha-2+2 \theta_{C} \geq 0$, see (4.6). The restriction $\theta_{C}<\frac{1}{2}$ in Theorem 3.4 then leads to $1-\frac{\alpha}{2} \leq \theta_{C}<\frac{1}{2}$, so that $\alpha>1$. On the other hand, in the case of Dirichlet boundary conditions one has $\partial W^{\alpha, p}(S)=W^{\alpha-\frac{1}{p}, p}(\partial S)$ and the Neumann map $N(t)$ has to be replaced by the Dirichlet map $D(t) \in \mathcal{B}\left(\partial W^{\alpha, p}(S), W^{\alpha, p}(S)\right)$, where $D(t) y:=x \in W^{\alpha, p}(S)$ is the solution of the elliptic problem

$$
\begin{aligned}
\mathcal{A}(t, \cdot, D) x=0 & \text { on } S, \\
x=y & \text { on } \partial S
\end{aligned}
$$

for a given $y \in \partial W^{\alpha, p}(S)$. To achieve that $\Lambda_{C}(t):=-A_{-1}(t) D(t)$ maps into $X_{-\theta_{C}}^{t}$, we need that $H^{\alpha, p}(S)=H_{\mathcal{B}(t)}^{\alpha, p}(S)$, and hence $\alpha-\frac{1}{p}<0$ in the Dirichlet case; which contradicts $\alpha>1$ and $p \geq 1$.

\section{REFERENCES}

[1] P. ACQUistaPACE, Evolution operators and strong solutions of abstract linear parabolic equations, Differential Integral Equations 1 (1988), no. 4, 433-457.

[2] P. Acquistapace And B. Terreni, A unified approach to abstract linear nonautonomous parabolic equations, Rend. Sem. Mat. Univ. Padova 78 (1987), 47-107.

[3] S. Albeverio and Yu. A. Rozanov, On stochastic boundary conditions for stochastic evolution equations, Teor. Veroyatnost. i Primenen. 38 (1993), no. 1, 3-19.

[4] E. Alòs And S. BonacCorsi, Stability for stochastic partial differential equations with Dirichlet white-noise boundary conditions, Infin. Dimens. Anal. Quantum Probab. Relat. Top. 5 (2002), no. 4, 465-481.

[5] H. Amann, Nonhomogeneous linear and quasilinear elliptic and parabolic boundary value problems, Function spaces, differential operators and nonlinear analysis (Friedrichroda, 1992), Teubner-Texte Math., vol. 133, Teubner, Stuttgart, 1993, pp. 9-126.

[6] H. Amann, Linear and quasilinear parabolic problems. Vol. I, Abstract linear theory, Monographs in Mathematics, vol. 89, Birkhäuser Boston Inc., Boston, MA, 1995.

[7] V. I. Bogachev, Gaussian measures, Mathematical Surveys and Monographs, vol. 62, American Mathematical Society, Providence, RI, 1998.

[8] Z. BrZeŹNIAK, On stochastic convolution in Banach spaces and applications, Stochastics Stochastics Rep. 61 (1997), no. 3-4, 245-295.

[9] Z. BRZEŹNIAK AND J.M.A.M. van NEeRven, Stochastic convolution in separable Banach spaces and the stochastic linear Cauchy problem, Studia Math. 143 (2000), no. 1, 43-74.

[10] Z. BRZEŹNIAK AND J.M.A.M. van NeERVEn, Space-time regularity for linear stochastic evolution equations driven by spatially homogeneous noise, J. Math. Kyoto Univ. 43 (2003), no. 2, 261-303.

[11] D. L. Burkholder, Martingales and singular integrals in Banach spaces, Handbook of the geometry of Banach spaces, Vol. I, North-Holland, Amsterdam, 2001, pp. 233-269.

[12] G. Da Prato and J. ZabCZYK, Ergodicity for infinite-dimensional systems, London Mathematical Society Lecture Note Series, vol. 229, Cambridge University Press, Cambridge, 1996.

[13] A. Debussche, M. Fuhrman, And G. Tessitore, Optimal control of a stochastic heat equation with boundary-noise and boundary-control, ESAIM Control Optim. Calc. Var. 13 (2007), no. 1, 178-205 (electronic).

[14] R. Denk, G. Dore, M. Hieber, J. Prüss, And A. Venni, New thoughts on old results of $R$. T. Seeley, Math. Ann. 328 (2004), no. 4, 545-583. 
[15] D. Di Giorgio, A. Lunardi, And R. Schnaubelt, Optimal regularity and Fredholm properties of abstract parabolic operators in $L^{p}$ spaces on the real line, Proc. London Math. Soc. (3) 91 (2005), no. 3, 703-737.

[16] J. Diestel, H. Jarchow, and A. Tonge, Absolutely summing operators, Cambridge Studies in Advanced Mathematics, vol. 43, Cambridge University Press, Cambridge, 1995.

[17] S. KWAPIEŃ AND W. A. WoyCZyŃSKi, Random series and stochastic integrals: single and multiple, Probability and its Applications, Birkhäuser Boston Inc., Boston, MA, 1992

[18] L. Maniar And R. Schnaubelt, The Fredholm alternative for parabolic evolution equations with inhomogeneous boundary conditions, J. Differential Equations 235 (2007), no. 1, 308339.

[19] L. Maniar and R. Schnaubelt, Robustness of Fredholm properties of parabolic evolution equations under boundary perturbations, J. Lond. Math. Soc. (2) 77 (2008), no. 3, 558-580.

[20] B. Maslowski, Stability of semilinear equations with boundary and pointwise noise, Ann. Scuola Norm. Sup. Pisa Cl. Sci. (4) 22 (1995), no. 1, 55-93.

[21] J.M.A.M. van Neerven, M.C. Veraar, and L.W. Weis, Stochastic integration in UMD Banach spaces, Ann. Probab. 35 (2007), no. 4, 1438-1478.

[22] J.M.A.M. van Neerven, M.C. VeraAr, And L.W. Weis, Stochastic evolution equations in UMD Banach spaces, J. Functional Anal. 255 (2008), 940-993.

[23] G. Pisier, Martingales with values in uniformly convex spaces, Israel J. Math. 20 (1975) no. $3-4,326-350$.

[24] R. Schaubelt And M.C. VeraAR, Structurally damped plate and wave equations with random point force in arbitrary space dimensions., submitted.

[25] R. Schnaubelt, Asymptotic behaviour of parabolic nonautonomous evolution equations, Functional analytic methods for evolution equations, Lecture Notes in Math., vol. 1855, Springer, Berlin, 2004, pp. 401-472.

[26] R. SEELEy, Interpolation in $L^{p}$ with boundary conditions, Studia Math. 44 (1972), 47-60.

[27] R. B. Sowers, Multidimensional reaction-diffusion equations with white noise boundary perturbations, Ann. Probab. 22 (1994), no. 4, 2071-2121.

[28] H. TRIEBEL, Interpolation theory, function spaces, differential operators, second ed., Johann Ambrosius Barth, Heidelberg, 1995.

[29] M.C. VERAAR, Non-autonomous stochastic evolution equations and applications to stochastic partial differential equations, to appear in Journal of Evolution Equations.

[30] A. YAGI, Parabolic evolution equations in which the coefficients are the generators of infinitely differentiable semigroups. II, Funkcial. Ekvac. 33 (1990), no. 1, 139-150.

[31] A. YAGI, Abstract quasilinear evolution equations of parabolic type in Banach spaces, Boll. Un. Mat. Ital. B (7) 5 (1991), no. 2, 341-368.

Institute of Analysis, Department of Mathematics, Karlsruhe Institute of Technology (KIT), D-76128 Karlsruhe, Germany

E-mail address: schnaubelt@math.uni-karlsruhe.de

Delft Institute of Applied Mathematics, Delft University of Technology, P.O. Box 5031, 2600 GA Delft, The Netherlands

E-mail address: m.c.veraar@tudelft.nl, mark@profsonline.nl 